\title{
GSM-Based Wireless Database Access For Food And Drug Administration And Control
}

\author{
Engr. Prof Hyacinth C. Inyiama \\ Electronic and Computer Engineering Dept \\ Nnamdi Azikiwe University \\ Awka, Nigeria \\ Engr. Dr. Mrs. Christiana C. Okezie \\ Electronic and Computer Engineering Dept \\ Nnamdi Azikiwe University \\ Awka, Nigeria
}

\author{
Engr. Mrs Lois Nwobodo \\ Electronic and Computer Engineering Dept \\ Nnamdi Azikiwe University \\ Awka, Nigeria \\ Engr. Mrs. Nkolika O. Nwazor \\ Research and Development Department \\ Electronic Development Institute \\ Awka, Nigeria
}

\begin{abstract}
GSM (Global system for mobile communication) based wireless database access for food and drug administration and control is a system that enables one to send a query to the database using the short messaging system (SMS) for information about a particular food or drug. It works in such a way that a user needs only send an SMS in order to obtain information about a particular drug produced by a pharmaceutical industry. The system then receives the SMS, interprets it and uses its contents to query the database to obtain the required information. The system then sends back the required information to the user if available; otherwise it informs the user of the unavailability of the required information. The application database is accessed by the mobile device through a serial port using AT commands. This system will be of great help to the National Agency for Food and Drug Administration and Control (NAFDAC), Nigeria in food and drug administration and control. It will help give every consumer access to information about any product he/she wants to purchase, and it will also help in curbing counterfeiting. NAFDAC can also use this system to send SMS alerts on banned products to users of mobile phones. Its application can be extended to match the needs of any envisaged user in closely related applications involving wireless access to a remote database.
\end{abstract}

Keywords- Web; GSM; SMS; AT commands; Database; NAFDAC.

\section{INTRODUCTION}

The National Agency for Food and Drug Administration and Control (NAFDAC), established by Decree No. 15 of 1993 as amended is a Parastatal of the Federal Ministry of Health, with the mandate to regulate and control quality standards for Foods, Drugs, Cosmetics, Medical Devices, Chemicals, Detergents and packaged water imported, manufactured locally and distributed in Nigeria[1]. The importance of food and drugs to man and animal is very obvious. They need healthy food in order to grow and sustain life. The organs of the body may not function properly if exposed to unhealthy food. These situations of ill health provide the compelling need for drugs in order to modify the functioning of the body and restore it to a healthy state. To be acceptable, the drug must not be deleterious to the body but should rather lead to the restoration of normal life. In like manner, cosmetics should have no harmful effect on the body to which they are applied. It is the duty of all government to protect the health of the citizens, and in Nigeria, this is the responsibility of the Federal Ministry of Health [2].

The NAFDAC management has made intensive efforts towards making positive impacts in the lives of stakeholders (consumers and Dealers) through public enlightenment campaigns, education, persuasions and prosecution of defaulters.

\section{Problem Statement}

Counterfeiting is a global problem. Many goods moving through international commerce are counterfeited. Industry data show that $5-7 \%$ of world trade, valued at about US $\$ 280$ billion is lost to counterfeiting. The pharmaceutical industry and the personal care products industry in Nigeria are riddled with counterfeits. Millions of dollars of counterfeit pharmaceuticals and personal care products are reported to move through various authorized and unauthorized channels. These (authorized and unauthorized) channels make it possible for counterfeits, expired, repackaged and relabeled products to be shipped internationally. Several criminal networks involved in drug faking and counterfeiting have evolved over the years. They include manufacturers, importers, distributors and retailers. Other collaborators are inspection agents, shipping and clearing agents and corrupt government officials of drug regulatory agencies, customs and police [3].

NAFDAC is established to do the following among other functions:

- Regulate and control the importation, exportation, manufacture, advertisement, distribution, sale and use of drugs, cosmetics, medical devices, bottled water and chemicals

- Conduct appropriate tests and ensure compliance with standard specifications designated and approved by the council for the effective control of quality of food, 
drugs, cosmetics, medical devices, bottled water and chemicals and their raw materials as well as their production processes in factories and other establishments.

- Undertake inspection of imported food, drugs, cosmetics, medical devices, bottled water and chemicals and establish relevant quality assurance system, including certification of the production sites and of the regulated products.

- Undertake the registration of food, drugs, medical devices, bottled water and chemicals

- Collaborate with the National Law Enforcement Agency in measures to eradicate drug abuse in Nigeria

NAFDAC have succeeded in registering most food and certified okay by it. These products are issued registration numbers for easy identification. This has helped in reducing counterfeits. They have also gone a long way in sensitizing most Nigerians on the need for a product to be certified okay by them, but there is still the challenge of some products bearing fake NAFDAC registration number. Information about fake or substandard products banned from the Nigerian market can easily be accessed at the organization's web site. The awareness about such products is also created over the media. But there is the challenge of making this information available to those in areas without internet access or coverage. Moreover, there is the problem of low internet literacy. The problem of poor energy supply being faced by Nigeria makes it difficult for a greater proportion of the populace to have access to this information dispersed over the media.

Telecommunication has turned the world into a global village. It is a very cheap means of communication and is available in most parts of Nigeria. Technology is made more easily affordable if what is available is in a society is used to meet the immediate needs of that society. The challenges faced by other means of communication like the satellite (via internet), television, etc., led us to the introduction of this system that makes use of short messaging system (SMS) which is very cheap. Moreover, the GSM network over which it is sent is easily available in many parts of the country.

\section{Schematic Overview Of The Main Components IN A GSM NETWORK}

The block diagram overview of a GSM network is shown in figure1.The GSM network consists mainly of the following functional parts [4]:

- $\quad \mathrm{MSC}$ - the mobile service switching centre (MSC) is the core switching entity in the network.

- VLR - the visitor location register (VLR) contains subscriber data for subscribers registered in an MSC. Every MSC contains a VLR. Although MSC and VLR are individually addressable, they are always contained in one integrated node.

- $\quad$ GMSC - the gateway MSC (GMSC) is the switching entity that controls mobile terminating calls. When a call is established towards a GSM subscriber, a GMSC contacts the HLR of that subscriber, to obtain the address of the MSC where the subscriber is currently registered. That MSC address is used to route the call to the subscriber.

- HLR - the home location register (HLR) is the database that contains a subscription record for each subscriber of the network. A GSM subscriber is normally associated with a particular HLR. The HLR is responsible for the sending of subscription data to the VLR (during registration) or GMSC (during mobile terminating call handling).

- $\mathrm{CN}$ - the core network $(\mathrm{CN})$ consists of, amongst other things, MSC(s), GMSC(s) and HLR(s). These entities are the main components for call handling and subscriber management.[5]

- $\mathrm{BSS}$ - the base station system (BSS) is composed of one or more base station controllers (BSC) and one or more base transceiver stations (BTS).

- MOBILE STATION (MS) - The mobile station is the user equipment in GSM. The MS is what the user can see of the GSM system. The station consists of two entities, the Mobile Equipment (the phone itself), and the Subscriber Identity Module (SIM), in form of a smart card contained inside the phone.

\section{A. Signaling in GSM}

The various entities in the GSM network are connected to one another through signaling networks. Signaling is used for subscriber mobility, subscriber registration, call establishment, etc. The connections to the various entities are known as 'reference points'. Examples as seen from figure1 include the following [6]:

- A interface - the connection between MSC and BSC

- Abis interface - the connection between BSC and BTS

- C interface- the connection between GMSC and HLR

- D interface - the connection between MSC and HLR

- Um interface - the radio connection between MS and BTS

- ISDN user part (ISUP) - ISUP is the protocol for establishing and releasing circuit switched calls.

\section{B. Mobile station}

The Mobile Station, i.e. the GSM handset, is logically built up from the following components:

- Mobile equipment (ME) - this is the GSM terminal, excluding the SIM card;

- Subscriber identification module (SIM) - this is the chip embedded in the SIM card that identifies a subscriber of a GSM network; the SIM is embedded in the SIM card. 


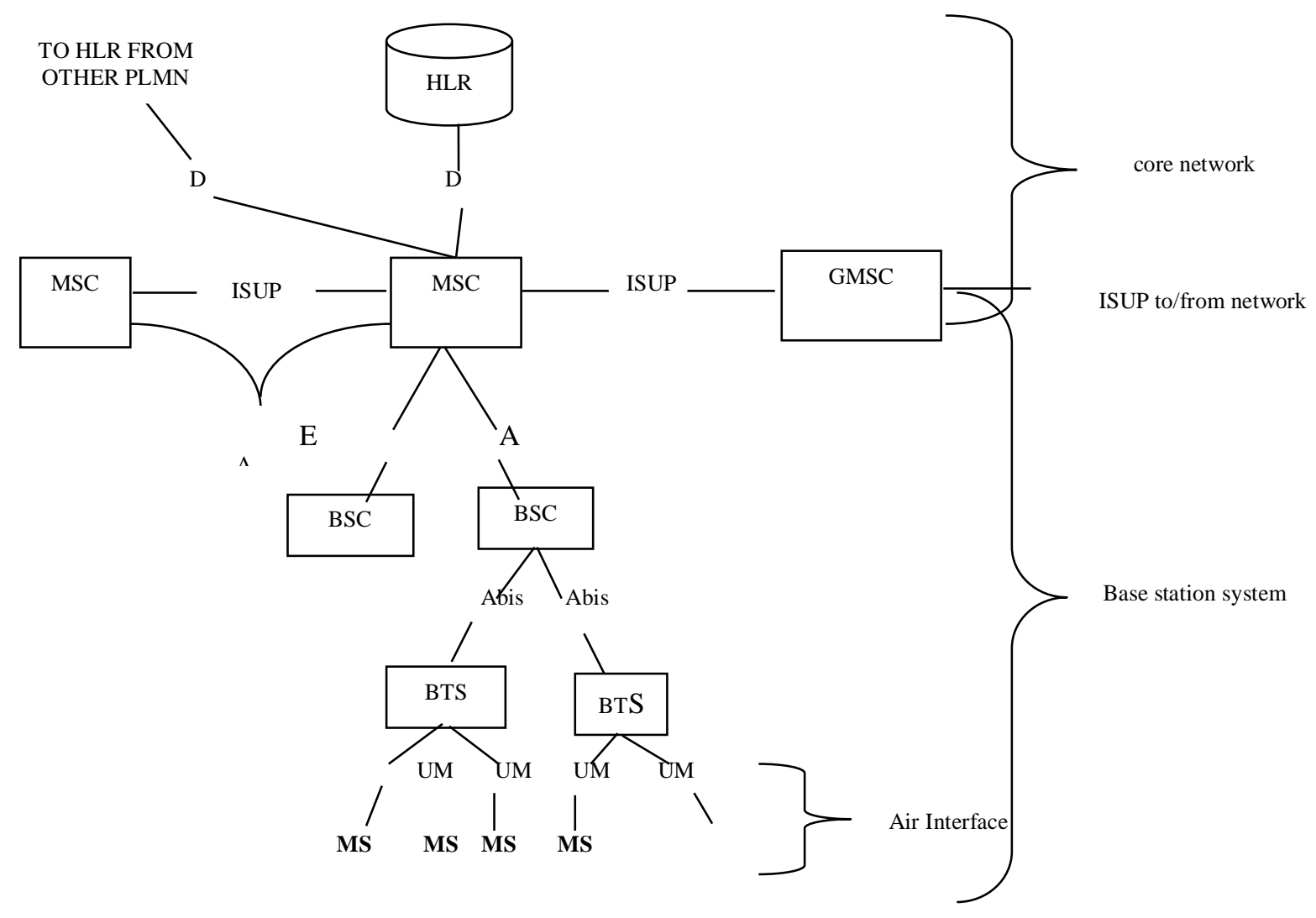

Figure 1. Overview of the GSM network

When the SIM card is inserted in the ME, the subscriber may register with a GSM network. The ME is now effectively personalized for this GSM subscriber.

\section{Short message service system (SMS)}

SMS is an acronym of Short Message Service, it is a communication service component a mobile communication systems, usually in a text form, [4] it uses standard rules which allows the exchange of short text messages between mobile phone devices, it could be from computer to phone or vice versa. SMS has used on modern handsets originated from radio telegraphy in radio memo pagers using standardized phone protocols. It was later defined as part of the Global System for Mobile Communications (GSM) series of standards in 1985 as a means of sending messages not more than 160 characters in a phone page, to and from GSM mobile handsets. GSM defines the Short Message Service - Cell Broadcast (SMS-CB), which allows messages (advertising, public information, etc.) to be broadcast to all mobile users in a specified geographical area. Messages are sent to a Short message service center (SMSC) which provides a "store and forward" mechanism. It attempts to send messages to the SMSC's recipients. If a recipient is not reachable, the SMSC queues the message for later retry. Some SMSCs also provide a "forward and forget" option where transmission is tried only once.

Both mobile terminated (MT, for messages sent to a mobile handset) and mobile originating (MO, for those sent from the mobile handset) operations are supported. Message delivery is usually "best effort", so there are no guarantees that a message will actually be delivered to its recipient, but delay or complete loss of a message is uncommon

\section{OPERATION OF The EXISTING System}

It is the duty of NAFDAC to register new drug, cosmetics, bottled water or drinks produced in Nigeria or imported from other countries which has successfully passed its tests. When these products are registered, a NAFDAC registration number is issued for the product which is written on the product's pack. Some manufacturers push their substandard products to the market without going through this process. NAFDAC officials physically visit some markets and pharmaceutical stores to ensure that the products being sold to consumers are approved by it. They take the record of these products and go back to their office to crosscheck if it is in their records.

\section{THE PROPOSED SYSTEM}

The proposed system is a system that will help users to access information on an item effectively, without wasting energy and time as far as there is a GSM network covering that area. The proposed system is aimed at achieving many things. It is an easy way of detecting fake products, or unregistered product. It increases productivity and ensures adequate management, ease of update and maintenance of operation, speed optimization and reduces the use of manual processing. It is reliable. System design in most cases are based on modularization, be it software or hardware systems. The project design would be based on modularization which is the divide and conquer method. 


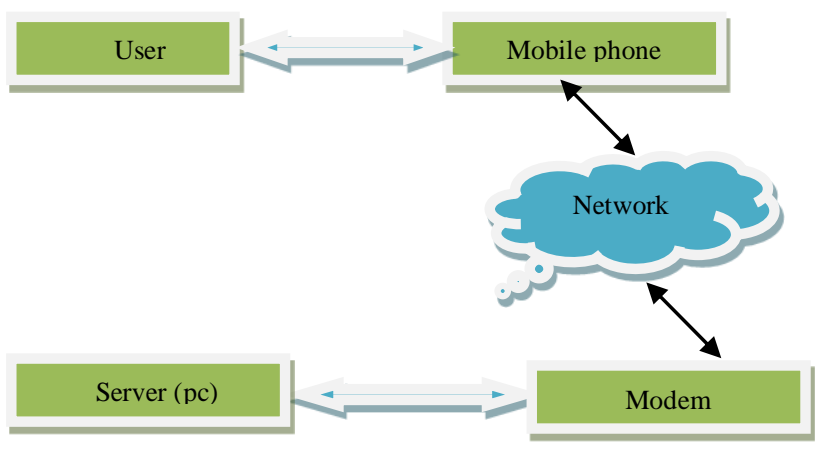

Fioure 2. Diaoram of the new svstem

It allows for easy and effective design as the whole system is broken down into smaller units or modules, and design for each module is carried out independently. Modularization facilitates easy troubleshooting and correctness or easy debugging (in the case of software development).

\section{SYSTEM SPECIFICATION}

This is the specification for the entire system. That is the various components integrated in the construction of a project as shown in Figure2. The processes of operation of the various parts of the system are as described hereunder.

The components of the system are:

- User's phone and internet modem.

- A server ( Personal Computer) running on Windows Operating System

- Protocol distribution unit (PDU)

- Database management system and Visual Basic Programming Environment

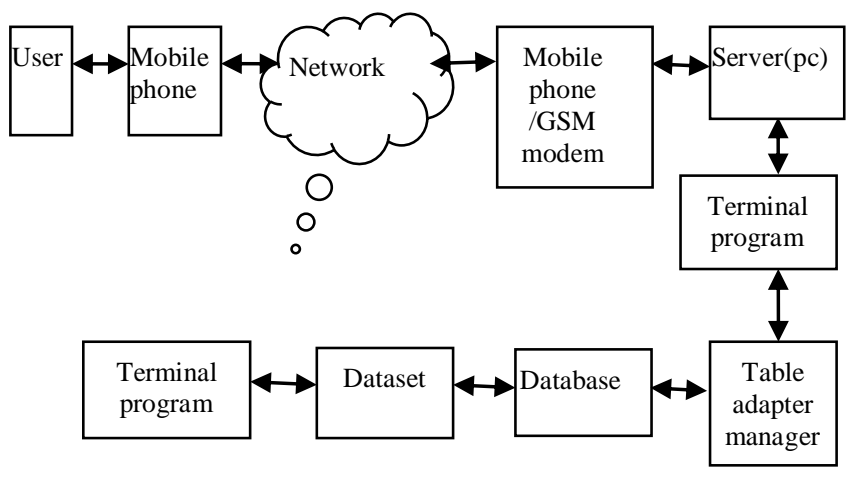

Figure 3. Block diagram representation of the operation of the system

Figure 3 shows the user who keys in the message and the message destination in a chosen format. The message format is of the form:

nafdac-regno-04-0437

Note: "nafdac" is the name of the table the system will query

$$
\begin{aligned}
& \text { "regno" is a search criteria } \\
& \text { "04-0437" is "the search with". }
\end{aligned}
$$

The message passes through the network, an object of the network known as the short message service system sends it to the owner, this object does the store and forward process, it stores the message if the recipient is not available on the network and forwards it if the recipient is available. The recipient in this system is the modem.

\section{A. Modem}

This is a device that modulates and demodulates, converting from analogue signal to digital signal, and vice versa. It facilitates easy transmission of signals from the mobile device to the server.

\section{B. Server}

It houses almost all the software components used in this project.

\section{Terminal program}

This instructs the computer on the next action to take when a message is received; it can be written in languages like Visual Basic, C\#, C++ and many others. For this system, visual basic.net was used.

\section{Table Adapter Manage}

Table Adapters provide communication between your application and a database. More specifically, a Table Adapter connects to a database, executes queries or stored procedures, and either returns a new data table populated with the returned data or fills an existing Data Table with the returned data. Table Adapters are also used to send updated data from your application back to the database.

\section{E. Database}

A database consists of an organized collection of data for one or more multiple uses.[7]

\section{F. Dataset}

The Data Set, which is an in-memory cache of data retrieved from a data source, it is a major component of the ADO.net architecture. The dataset is a memory where the Table Adapter keeps the retrieved data from the data source, when instructed by the terminal program.

\section{Software Subsystems Design}

\section{A. AT (Attention) Command}

The system uses AT COMMAND to establish communication with the modem. The AT commands that were used in this project are;

- AT+CGMS - Sends SMS Messages.

- $\mathrm{AT}+\mathrm{CMGD}$ - $\quad$ to clear the SMS receiving memory location in the GSM modem or message storage. 
- $\quad$ ATEO - makes the phone not to echo commands.

- $\mathrm{AT}+\mathrm{CPMS}$ - used to assign the message storage to read from, delete and write to.

- $\quad \mathrm{AT}+\mathrm{CMGR}$ - read SMS messages from storage.

- $\mathrm{AT}+\mathrm{CMGF}$ - tells the modem to operate in test mode.

B. Syntax for Sending SMS

The syntax for sending SMS to NAFDAC is shown below nafdac-drugname-puriton nafdac-sachetwater-aquarapha nafdac-regno-04-0437

\section{The SQL Command of NAFDAC Table}

FillGetdata( )

a) fillBy- drug name,GetDataby-drug name(drug name) then the SQL command is thus select drug name from nafdac where drug name $=$ ?

b) fillBy-sachet water,GetDataby- sachet water (sachet water)

then the SQL command is thus select sachet water from nafdac where satchet water $=$ ?

c) fillBy- reg no,GetDataby-reg no(reg no) then the SQL command is thus select reg no from library where reg no $=$ ?

\section{CONCLUSION}

The SMS based NAFDAC Registration Exercise is a new innovation that unveils the features of SMS in GSM applications. This work is a new development that will help curb the menace of counterfeit drugs in our society. This work can be easily adapted to match the need of any envisaged user.

\section{REFERENCES}

[1] http://www.nafdacnigeria.org. retrieved 22nd February 2011

[2] About NAFDAC. http://www.nafdacnigeria.org/about.html. retrieved 22nd february 2011

[3] Global Trends. http://www.nafdacnigeria.org/globaltrends.htm retrieved 22nd february 2011.

[4] S. Guthery, M. Cronin, Mobile Application Development with SMS and
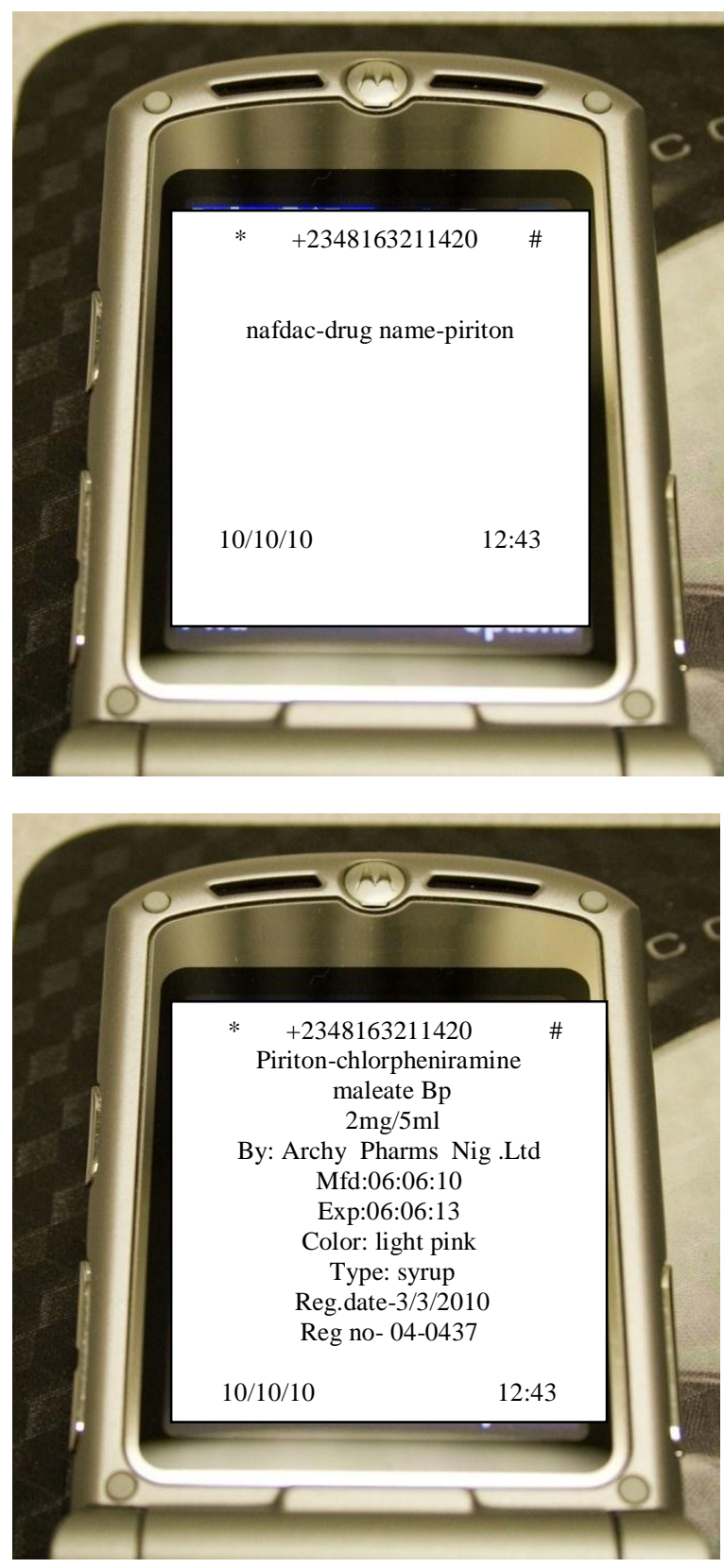

Figure 4. The Project Input and the Output

the SIM Toolkit, McGraw-Hill Professional,1997. Inc(2009) pp.5-10

[5] F. Hillebrand, F. Trosby, K. Holley \& I. Harrris, Short Message Service, Trafford Publishing, 2010, Page 19.

[6] J.Eberspaecher, H. J. Voegel, C. Bettstetter, GSM Switching, Services, And Protocols, John Wiley \& Sons,2001, Pg 254-267

[7] C.J Date,An Introduction to database systems, Wiley, 1998, pg 1. 